\title{
Embracing sensorimotor history: Time-synchronous and time-unrolled Markov blankets in the free-energy principle
}

\author{
Nathaniel Virgo ${ }^{1}$, Fernando Rosas ${ }^{2,3,4}$, Martin Biehl $^{5}$ \\ nathanielvirgo@elsi.jp, f.rosas@imperial.ac.uk, martin.biehl@cross-compass.com \\ ${ }^{1}$ Earth-Life Science Institute (ELSI), Tokyo Institute of Technology, Japan \\ ${ }^{2}$ Centre for Psychedelic Research, Department of Brain Science, \\ Imperial College London, UK \\ ${ }^{3}$ Data Science Institute, Imperial College London, UK \\ ${ }^{4}$ Centre for Complexity Science, Imperial College London, UK \\ ${ }^{5}$ Cross Labs, Cross Compass, Japan
}

This paper is a commentary on Bruineberg, J., Dolega, K., Dewhurst, J., and Baltieri, M. (2021). The Emperor's New Markov Blankets. Behavioral and Brain Sciences. https://doi.org/10.1017/S0140525X21002351

\begin{abstract}
The free-energy principle (FEP) builds on an assumption that sensormotor loops exhibit Markov blankets in stationary state. We argue that there is rarely reason to assume a system's internal and external states are conditionally independent given the sensorimotor states, and often reason to assume otherwise. However, under mild assumptions internal and external states are conditionally independent given the sensorimotor history.
\end{abstract}

Bruineberg and colleagues provide a thorough review of Markov blankets and their limitations in the context of the free-energy principle (FEP). We wish to complement this by drawing attention to two additional issues that we believe have important consequences for the FEP. Firstly, contrary to what one might expect, the condition known as "Markov blanket" in the FEP literature is generally not guaranteed by a sensor-motor loop structure. Secondly, the Markov blanket condition needed for the FEP is far stronger than it appears to be. These issues severely limit the scope of applicability of current formulations of the FEP. Fortunately, we believe they can be solved, and give some hints towards a resolution.

As Bruineberg et al. explain, the notion of a Markov blanket arises in the context of graphical models, and in particular, Bayesian networks. In a Bayesian network each node represents a random variable, and their joint distribution 


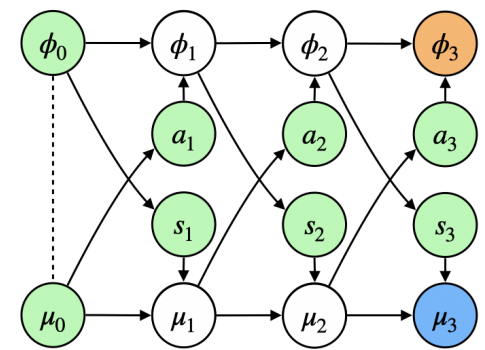

Figure 1: top: A "time-unrolled" sensor-motor loop in discrete time. The time-unrolled graph is a Bayesian network, and hence the Pearl framework can be applied. It follows that the current internal and external states, $\mu_{3}$ and $\phi_{3}$ (blue, orange) are conditionally independent given the nodes in light green, which consist of the past histories of sensor and actuator states, $s_{1}, s_{2}, s_{3}$ and $a_{1}, a_{2}, a_{3}$, as well as the initial states $\mu_{0}$ and $\phi_{0}$. (The dashed line indicates that the initial states might be correlated.) bottom: The current internal and external

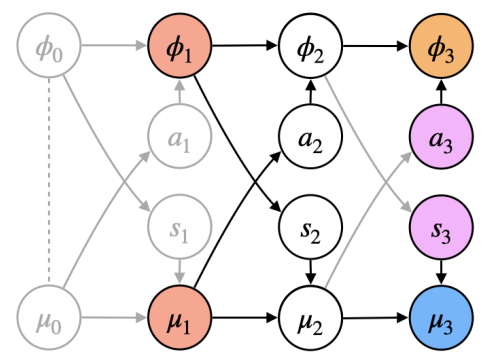
states are in general not conditionally independent given only the current sensor and actuator states, $s_{3}$ and $a_{3}$, because $\mu_{3}$ and $\phi_{3}$ have common ancestors that are not screened off by these nodes, e.g. $\mu_{1}$ and $\phi_{1}$ (red). This is true regardless of stationarity. If the system is ergodic then the dependence on the initial states will disappear in the infinite limit, so that we can effectively say that $\mu_{t}$ and $\phi_{t}$ are conditionally independent given the infinite past history of the sensorimotor states.

factors in a particular way that depends on the topology of the graph (Pearl 1988).

The literature on FEP is also concerned with graphs that are not Bayesian networks. Each node in these graphs represents a dynamical variable of a system and an edge represents the possibility that one dynamical variable can influence another. These include the adjacency matrix described in Bruineberg et al.'s Section 4.2, and also the sensor-motor loop as illustrated in their Figure 2. Typically, the edges in such graphs correspond to nonzero terms in a Jacobian matrix. We will call such graphs influence graphs.

A stationary state defines a joint distribution over the nodes of an influence graph. There is then some resemblance between the influence graphs and Bayesian networks, since both contain nodes that represent random variables and edges that represent influences of some kind. However, these two types of graph are fundamentally different. Influence graphs are not necessarily acyclic, but more importantly, the theorems in Pearl's formalism do not apply to influence graphs. In particular, one might expect that the sensor-motor loop (Bruineberg et al.'s Figure 2) would imply the time-synchronous Markov blanket condition

$$
\mu_{t} \Perp \phi_{t} \mid s_{t}, a_{t} .
$$

However, this is not the case in general - and this is important because (1) is used in deriving the FEP. This issue was recently pointed out (Biehl et al., 2021; Aguilera et al. 2021), and while it has been acknowledged in some of the most recent FEP literature it is not as widely known as it should be. We sketch the underlying reason for it in Figure 1. 
Recent works (e.g. Friston et al., 2021a; Friston et al., 2021b) have sought to address this by seeking additional conditions or conjectures under which the needed relationship holds. However, the fact that these conditions are highly non-trivial suggest that the scope of the FEP may be much more limited than previously thought.

Furthermore, (1) itself puts a very strong constraint on a system's dynamics. One way to see this is via the data processing inequality (Cover and Thomas, 2006 , p. 34), which imposes that if (1) holds then all information that $\mu_{t}$ and $\phi_{t}$ share needs to be present in $\left(s_{t}, a_{t}\right)$. This would mean that the internal and external states could share no more information than is contained in the sensor and motor states at the current time.

But cases where information is stored in the environment and the agent but not in the blanket are ubiquitous. Imagine a friend gives you a phone number written on a piece of paper, which you memorise and then store in a box. The statistical independence between internal and external variables conditioned on active and sensory ones is broken as soon as the piece of paper is away from your sensory input. Once it's out of sight the phone number cannot be stored simultaneously in your internal state and on the piece of paper. As Parr et al. (2021) discuss, this need not be true in transients even if it holds in stationary state. Nevertheless it puts an unrealistic constraint on the stationary dynamics, which we don't expect to be applicable to living organisms.

A possible resolution of this limitation follows from Figure 1. Although (1) cannot be assumed for a general sensor-motor loop, we $d o$ have the relationship

$$
\mu_{t} \Perp \phi_{t} \mid s_{t}, a_{t}, s_{t-1}, a_{t-1}, s_{t-2}, a_{t-2}, \ldots .
$$

We expect an analogous result in continuous time. The internal and external states are not conditionally independent given the current sensorimotor states, but, under only mild assumptions, they are conditionally independent given the sensorimotor history. Alternative constructions of blankets that follow these principles are currently being investigated (e.g. Rosas et al. 2020).

This makes intuitive sense: your knowledge of the world is not limited by what you can sense at the current moment, but it is limited by what you have been able to sense over your whole lifetime. If a new version of the FEP can be constructed based on this alternative conditional independence relation, then it will be more encompassing and will have something close to the broad applicability that was originally intended.

\section{Funding statement}

M.B. and N.V. acknowledge the support of Grant 62229 from the John Templeton Foundation. The opinions expressed in this publication are those of the author(s) and do not necessarily reflect the views of the John Templeton Foundation. F.R. is supported by the Ad Astra Chandaria Foundation. 


\section{Conflicts of interest statement}

None.

\section{References}

Aguilera, M., Millidge, B., Tschantz, A., and Buckley, C. L. (2021). How particular is the physics of the free energy principle? Physics of Life Reviews.

Biehl, M., Pollock, F. A., \& Kanai, R. (2021). A technical critique of some parts of the Free Energy Principle. Entropy, 23(3), 293.

Cover, T. M. \& Thomas, J. A. (2006). Elements of information theory (second edition). John Wiley \& Sons.

Friston, K. J., Da Costa, L., \& Parr, T. (2021a). Some interesting observations on the free energy principle. Entropy, 23(8), 1076.

Friston, K., Heins, C., Ueltzhöffer, K., Da Costa, L., \& Parr, T. (2021b). Stochastic Chaos and Markov Blankets. Entropy, 23(9), 1220.

Parr, T., Da Costa, L., Heins, C., Ramstead, M. J. D., \& Friston, K. J. (2021). Memory and Markov Blankets. Entropy, 23(9), 1105.

Pearl, J. (1988). Probabilistic reasoning in intelligent systems: networks of plausible inference. Morgan Kaufmann.

Rosas, F. E., Mediano, P. A., Biehl, M., Chandaria, S., \& Polani, D. (2020, September). Causal blankets: Theory and algorithmic framework. In International Workshop on Active Inference (pp. 187-198). Springer, Cham. 